\title{
Correction to: 2D and 3D image localization, compression and reconstruction using new hybrid moments
}

\author{
Mohamed Amine Tahiri ${ }^{1}$ Hicham Karmouni ${ }^{1}$ (D) Mhamed Sayyouri ${ }^{2}$. \\ Hassan Qjidaa ${ }^{1}$
}

Published online: 2 March 2022

๑) Springer Science+Business Media, LLC, part of Springer Nature 2022

\section{Correction to: Multidimensional Systems and Signal Processing https://doi.org/10.1007/s11045-021-00810-y}

The original version of this article unfortunately contained a mistake. In this article the affiliation details for Author Mhamed Sayyouri were incorrectly given as 'Laboratory of Engineering Sciences for Energy LabSIPE, National School of Applied Sciences of ElJadida, Chouaïb Doukkali University, PO Box 1166, 24,004 EL Jadida Plateau, Morocco' but should have been 'Laboratory of Engineering, Systems and Applications, National School of Applied Sciences, Sidi Mohamed Ben Abdellah University, BP 72, My Abdallah Avenue Km. 5 Imouzzer Road, Fez, Morocco'.

The original article has been corrected.

Publisher's Note Springer Nature remains neutral with regard to jurisdictional claims in published maps and institutional affiliations.

The original article can be found online at https://doi.org/10.1007/s11045-021-00810-y.

$凶$ Hicham Karmouni

hicham.karmouni@usmba.ac.ma

Mohamed Amine Tahiri

mohamedamine.tahiri@usmba.ac.ma

Mhamed Sayyouri

mhamed.sayyouri@usmba.ac.ma

Hassan Qjidaa

qjidah@yahoo.fr

1 Laboratory of Electronic Signals and Systems of Information LESSI, Dhar El Mahrez Faculty of Science, CED-ST, STIC, Sidi Mohamed Ben Abdellah-Fez University, Fez, Morocco

2 Laboratory of Engineering, Systems and Applications, National School of Applied Sciences, Sidi Mohamed Ben Abdellah University, BP 72, My Abdallah Avenue Km. 5 Imouzzer Road, Fez, Morocco 